\title{
Quando la carenza di ferro diventa rara...
}

\author{
Marianna Casertano, Nicoletta Di Maio, Angela Maria Caprio, Maddalena Casale, \\ Sofia MR Matarese, Silverio Perrotta \\ Ematologia e Oncologia Pediatrica, Dipartimento della Donna, del Bambino e di Chirurgia Generale e Specialistica, \\ Azienda Dipartimento della Donna, del Bambino e di Chirurgia Generale e Specialistica, Azienda Ospedaliera \\ Universitaria-Università degli Studi di Napoli Luigi Vanvitelli, Napoli
}

L'anemia sideropenica è il disordine nutrizionale più comune al mondo, la forma di anemia globalmente più diffusa e sicuramente la più frequente in età pediatrica. In presenza di un'anemia microcitica, l'evidenza di una carenza di ferro all'assetto marziale (indice di saturazione della transferrina $<10 \%$ e ferritina $<12 \mathrm{mg} / \mathrm{l}$ ) consente la diagnosi. Le cause più comuni di carenza marziale sono un aumento delle richieste e un ridotto introito, tipiche della prima infanzia e dell'adolescenza. È necessario assicurarsi della correzione del disordine a fine terapia, per poter escludere cause più rare di carenza di ferro, è necessario garantire ai pazienti una corretta diagnosi e terapia, risparmiando così risorse economiche e stress per l'intera famiglia.

Sono qui descritti due casi di anemia ferrocarenziale dalla eziopatogenesi molto diversa e il percorso che ha portato alla diagnosi.

\section{Iron deficiency anemia is the most common nutritional disorder in the world, the most widespread form of anemia and the most frequent in children. In the presence of microcytic anemia, evidence of an iron deficiency (transferrin saturation index $<10 \%$ and ferritin $<12 \mathrm{mg} / \mathrm{ll}$ ) allows the diagnosis. An increase in requests and a reduced income are the most common causes in early childhood and adolescence. It is essential to ensure that the disorder is corrected at the end of therapy, in order to exclude rarer causes of iron deficiency. A correct diagnosisi and therapy are also essential to guarantee patients so to spare additional costs and stress for the whole family.}

\section{La storia}

\section{Caso 1}

Giunge alla nostra attenzione A., 7 anni. In anamnesi una storia di anemia sideropenica cronica. Il primo riscontro di anemia microcitica-sideropenia avveniva circa 5 anni prima, durante un ricovero per gastroenterite all'età di 2 anni. La piccola in quell'occasione veniva sottoposta a workup diagnostico per escludere le cause più frequenti di anemia sideropenica ma tutto risultava negativo. Nonostante la terapia con numerosi cicli di ferro per via orale, l'anemia e la carenza marziale persistevano. Cominciava così una lunga serie di ricoveri, indagini e consulenze specialistiche che si concludevano sempre con una nuova supplementazione di ferro. Ripetuti gli esami ematochimici di primo livello, l'esame emocromocitometrico confermava l'anemia microcitica e l'assetto marziale evidenziava la carenza di ferro ( $\mathrm{Hb} 7,1 \mathrm{~g}$ ) dl; MCV 54,3 fl; reticolociti 36.200; indice di saturazione della transferrina (IST) $4 \%$; ferritina $18 \mathrm{ng} / \mathrm{dl}$ ). Si procedeva quin- di con un ulteriore work-up diagnostico per valutare un malassorbimento e/o una perdita di ferro. Sierologia per celiachia, ricerca di sangue occulto, coprocoltura, parassitologico, più volte ripetuto, calprotectina fecale e antigene per Helicobacter Pylori (HP) su feci risultavano tutti negativi. Il test da carico orale di ferro evidenziava un mancato assorbimento. S'iniziava, quindi, terapia marziale per via endovenosa, ma anche in questo caso il quadro ematologico non migliorava. Il quadro di anemia ferrocarenziale refrattaria alla terapia marziale sia per via orale che endovenosa induceva il forte sospetto di una forma genetica rara di anemia sideropenica.

\section{Caso 2}

V., 9 anni, veniva visto in ambulatorio per anemia sideropenica persistente.

Il primo riscontro avveniva diversi anni prima ma la causa dell'anemia non era stata mai identificata e il piccolo praticava da anni ripetuti cicli di terapia con diverse formulazioni di ferro orale. L'anamnesi familiare era negativa per patologie ema- tologiche. Al controllo veniva confermata l'anemia da carenza di ferro $(8,9 \mathrm{~g} / \mathrm{dl}$; MCV 64,5 fl; reticolociti 30.000; IST 6\%; ferritina $6 \mathrm{ng} / \mathrm{dl})$. Si valutavano le cause di malassorbimento e/o perdita di ferro che risultavano tutte negative (ricerca di sangue occulto, coprocoltura, parassitologico, calprotectina, antigene per Helicobacter Pylori su feci e sierologia per celiachia). Il test da carico orale di ferro era nella norma. Si decideva di praticare nuovamente terapia marziale per via orale a dosaggio adeguato, istruendo bene la madre alla corretta somministrazione del ferro. Al follow-up i valori dell'emoglobina miglioravano, ma non si normalizzavano e non si raggiungeva una normalizzazione dei depositi di ferro. Considerando la risposta incompleta, si intraprendeva una terapia con ferro per via endovenosa, che determinava una normalizzazione dei valori d'emoglobina e dell'assetto marziale ma con ricaduta rapida alla sospensione della terapia endovenosa. Il quadro clinico e la risposta alla terapia marziale orientavano verso una forma di anemia ferrocarenziale da aumentato fabbisogno di ferro.

\section{I percorsi diagnostici}

Caso 1. La mancata risposta a una terapia marziale sia per via orale che endovenosa deve indirizzare verso forme di anemia sideropenica da alterato metabolismo del ferro [1-2]. La sindrome IRIDA è una forma di anemia su base genetica, dovuta alla mutazione del gene TMPRSS6, presente sul cromosoma 22. Tale gene codifica per una serinproteasi trasmembrana prodotta a livello epatico, la Matriptasi2, che modula negativamente l'espressione dell'epcidina. Questa è un regolatore del metabolismo del ferro. Questo peptide prodotto a livello epatico inibisce una proteina transmembrana, la ferroportina, presente sulla superficie basolaterale degli enterociti intestinali e sulla membrana macrofagica, determinando un mancato 
assorbimento di ferro a livello intestinale e un mancato rilascio in circolo del ferro già presente nell'organismo da parte delle cellule del sistema reticolo-endoteliale. Mutazioni a carico del gene TMPRSS6, coinvolto nella sindrome IRIDA, determinano un aumento dell'epcidina e quindi un'inibizione della ferroportina. Dal punto di vista clinico si determina un'anemia moderata-grave e persistente, con $\mathrm{MCV}$ molto basso (45-65 fl), iposideremia grave (IST <5\%), risposta assente alla terapia marziale per via orale, incompleta e transitoria alla terapia marziale endovenosa. Nonostante la carenza di ferro grave, i pazienti con IRIDA presentano un normale sviluppo psicofisico e scarsi segni e sintomi legati all'anemia. Il quadro clinico tende a migliorare con la crescita per la progressiva riduzione del fabbisogno di ferro. La diagnosi di certezza si fonda sull'indagine molecolare del gene TMPRSS6. Attualmente i pazienti vengono trattati con ferro per via orale e acido ascorbico, che sembra migliorare l'assorbimento del ferro stesso. Si predilige la terapia orale poiché l'alterato metabolismo del ferro ne potrebbe determinare un accumulo a livello delle cellule del sistema reticolo endoteliale [3].

Caso 2. Nel caso di un'anemia sideropenica scarsamente responsiva alla terapia marziale per via orale, bisogna sempre considerare la possibilità che si tratti di un'infezione da HP, anche in un paziente senza sintomi gastrointestinali. Dal punto di vista fisiopatologico più meccanismi intervengono nella genesi dell'anemia, tra cui l'acloridria in presenza di gastrite e l'utilizzo del ferro per la crescita batterica. Il microrganismo, infatti, utilizza il ferro legato alla lattoferrina, che, in corso di infezione, aumenta. I sintomi di infezione da HP in età pediatrica sono aspecifici e possono includere dolore epigastrico, nausea e/o vomito, anoressia, ematemesi o anemia ferrocarente come unico segno. La diagnosi nel bambino deve essere sempre condotta attraverso test invasivi, quindi esofagogastroduodenoscopia (EGDS) con annessa ricerca dell'HP. Una prima valutazione, nel caso di anemia sideropenica non responsiva alla terapia, in cui siano state escluse altre cause di sideropenia, può essere effettuata con test non invasivi (come nel nostro caso la ricerca dell'antigene HP su feci). Va comunque considerato che, nel caso in cui i test non invasivi siano inconcludenti e l'anemia, pur correttamente trattata, persista o recidivi, è indicata l'EGDS con biopsia. Per la diagnosi è necessaria la positività della ricerca microscopica sulla biopsia (antro e corpo) associata alla positività di un secondo test (ureasi o coltura microbiologica). La terapia eradicante migliora significativamente i livelli di emoglobina, talvolta senza supplemento di ferro, risolvendo stabilmente il quadro ematologico.

\section{Commento}

A differenza di altre patologie ematologiche che possono presentarsi come eventi acuti e gravi per cui sono rapidamente riconosciute [4-8], l'anemia ferrocarenziale è un disordine cronico che tuttavia può presentare un notevole carico gestionale per controlli ripetuti, richieste di visite specialistiche, esami e terapie cicliche [9]. Il principale problema della gestione dell'anemia ferrocarenziale è legato all'individuazione dell'eziologia e alla correzione duratura del disordine. È fondamentale assicurare una corretta supplementazione con il ferro, in quanto errori nell'assunzione della terapia marziale o scarsa compliance a essa sono le cause principali della persistenza della carenza di ferro. Il controllo a fine terapia è fondamentale per definire la completa risoluzione del quadro clinico o la necessità di procedere con ulteriori indagini [10].

I fattori più comuni nella patogenesi della carenza marziale sono:

- Inadeguato apporto/aumentato fabbisogno: è quello che accade più frequentemente nel bambino. I momenti più critici sono il primo anno di vita $\mathrm{e}$ l'epoca puberale, per il rapido accrescimento. La carenza di ferro nel primo anno di vita è generalmente provocata da un allattamento materno esclusivo protratto oltre i sei mesi, da una prematurità e/o gemellarità; durante la crescita da diete inadeguate o malnutrizione.

- Ridotto assorbimento di ferro: tra le cause più frequenti vanno indagate celiachia, malattie infiammatorie croniche dell'intestino, gastrite cronica autoimmune $\mathrm{o}$, più raramente, infezione da HP.

- Aumentata perdita di ferro: con perdita di sangue evidente (come nel caso di mestruazioni abbondanti in giovani adolescenti) oppure occulta, generalmente gastrointestinale come nel caso di allergia alle proteine del latte vaccino nel primo anno di vita, ernia iatale, diverticolo di Meckel, parassitosi.

Se gli esami di primo livello non invasivi non sono chiarificatori, la risposta alla terapia marziale è l'elemento che deve sempre indirizzare l'iter diagnostico [10].

\section{Dai casi e dalla letteratura abbiamo im- parato che:}

- In caso di carenza di ferro, il primo livello diagnostico deve prevedere, oltre all'esame emocromocitometrico, l'assetto marziale completo ovvero sideremia, transferrina e ferritina. Spesso i bambini devono ripetere il prelievo perché la sideropenia è stata valutata solo con la sideremia o la ferritina.

- Va sempre rivalutata la risposta alla terapia marziale, che è il punto di arrivo del percorso diagnostico-terapeutico, quando si ottiene normalizzazione dei parametri ematologici e dell'assetto marziale, mentre è punto di partenza di un percorso diagnostico, nel caso in cui non vi sia adeguata risposta.

- La prima e più importante causa di fallimento della terapia marziale è la scorretta modalità di assunzione del ferro. Vanno sempre richieste e rivalutate le modalità di assunzione del ferro, come l'assunzione a digiuno, lontano dal latte o dal tè.

- Solo in caso di effettiva refrattarietà o incompleta risposta alla corretta terapia marziale, è necessario ricorrere all'ematologo pediatra e si deve prevedere il coinvolgimento del gastroenterologo pediatra per eventuali esami strumentali di II e III livello, essendo le patologie gastroenterologiche le cause più frequenti di mancata risposta alla terapia marziale, quando correttamente assunta.

- Vi sono infine da considerare le cause genetiche che, sebbene molto rare, devono essere considerate in presenza di reali fallimenti terapeutici ripetuti e dopo aver escluso tutte le altre cause di insuccesso terapeutico, al fine di stabilire il miglior approccio farmacologico specifico per quel paziente.

maddalena.casale@unicampania.it

La bibliografia è consultabile online. 
1. Hershko C, Camaschella C. How I treat unexplained refractory iron deficiency anemia. Blood 2014;16;123:326-33. doi: 10.1182/blood-2013-10-512624. Epub 2013 Nov 8. PMID: 24215034.

2. Casale M, Borriello A, Scianguetta S et al. Hereditary hypochromic microcytic anemia associated with loss-offunction DMT1 gene mutations and absence of liver iron overload. Am J Hematol 2018;93:E58-E60. doi: 10.1002/ajh.24988. Epub 2017 Dec 8. PMID: 29178181

3. Camaschella C, Nai A, Silvestri L. Iron metabolism and iron disorders revisited in the hepcidin era. Haematologica 2020;105:260-72. doi: 10.3324/haematol.2019.232124. PMID: 31949017; PMCID: PMC7012465.

4. Franchini M, Forni GL, Marano G et al. Red blood cell alloimmunisation in transfusion-dependent thalassaemia: a systematic review. Blood Transfus 2019;17:4-15. doi: 10.2450/2019.0229-18. PMID: 30653458; PMCID: PMC6343597.

5. De Franceschi L, Lux C, Piel FB et al.Access to emergency departments for acute events and identification of sickle cell disease in refugees. Blood 2019;133:2100-3. doi: 10.1182/blood-2018-09-876508. Epub 2019 Feb 11. PMID: 30745303.

6. Casale M, Russo G, Sainati L et al. La gestione del rischio infettivo nei bambini asplenici: raccomandazioni dal network italiano asplenia. Quaderni acp. 2019;4:158-61

7. Inusa B, Casale M, Booth C, Lucas S. Subarachnoid haemorrhage and cerebral vasculopathy in a child with sickle cell anaemia. BMJ Case Rep 2014;2014:bcr2014205464. doi: 10.1136/bcr-2014-205464. PMID: 25336550; PMCID: PMC4208269.

8. Casale M, Picariello S, Corvino F. Life-Threatening Drug-Induced Liver Injury in a Patient with $\beta$-Thalassemia Major and Severe Iron Overload on Polypharmacy. Hemoglobin 2018;42:213-6.

9. Camaschella C. Iron deficiency. Blood 2019;133:30-9. doi: 10.1182/blood-2018-05-815944. Epub 2018 Nov 6. PMID: 30401704.

10. Camaschella C. Iron-deficiency anemia. N Engl J Med 2015;372:1832-43. doi: 10.1056/NEJMra1401038. PMID: 25946282. 